\title{
THE ENVIRONMENTS OF RADIO GALAXIES
}

\author{
J.E. PESCE ${ }^{1}$, R. FALOMO ${ }^{2}$, G. FASANO ${ }^{2}$, AND R. SCARPA $^{3}$ \\ ${ }^{1}$ STScI, 3700 San Martin Dr., Baltimore, MD 21218 USA \\ ${ }^{2}$ OAP, vicolo dell'Osservatorio 5, 35122 Padova, Italy \\ ${ }^{3}$ Univ. di Padova, vicolo dell'Osservatorio 5, Padova, Italy
}

\section{Introduction}

In the unified schemes of AGNs BL Lac objects are believed to be FanaroffRiley (FR) type I radio galaxies with a relativistic jet aligned to the observer's line of sight (e.g. Urry \& Padovani 1995). Kollgaard et al. (1992) and Owen et al. (1995) suggest some FR II sources can also be BL Lac parents. Clearly, isotropic properties such as the galaxy environment of both beamed and unbeamed objects should be identical.

We are currently studying BL Lac environments (e.g. Pesce et al. 1995) and plan to compare them to those of radio galaxies. Previously, based on statistical analysis of sources at $\langle z\rangle=0.05$, Prestage \& Peacock (1988) concluded that FR I galaxies inhabit richer clusters than FR II galaxies.

Higher redshift samples were studied by Yates et al. $(1989 ;\langle z\rangle \sim 0.49)$ and Hill \& Lilly $(1991 ;\langle z\rangle \sim 0.45)$. These FR I and II galaxies are found in similar environments. For FR Is there is no change in environment with redshift. Few objects are found in clusters richer than Abell class 1.

These samples are not complete, however, and the analyses were inhomogeneous. We thus carried out a study of environments and host galaxies of a large sample of FR I and II sources. To date we analyzed 29/95 objects with $\langle z\rangle \sim 0.07$ selected from Wall \& Peacock (1985) and Ekers et al. (1989). Fasano et al. (1995) discuss the properties of this sample.

\section{Analysis}

We use the digitized ESO-SERC survey plates (cf. Lasker et al. 1990). The FOCAS software (Valdes 1982) was used to detect and classify all objects around the target. We calculate $\mathrm{N}_{0.5}$, the number of galaxies within 0.5 Mpc of the target with $m \leq m_{3}+2$ where $m_{3}$ is the 3rd brightest (cluster) galaxy, corrected for background (Bahcall 1981). 

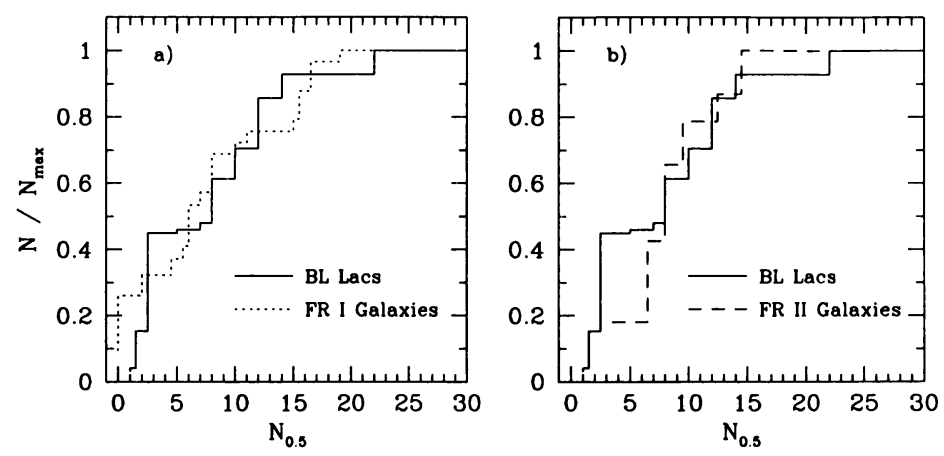

Figure 1. (a) The cumulative distribution of $\mathrm{N}_{0.5}$ for BL Lac objects (solid line; 11 objects) compared to that of FR I galaxies (dotted line; 21 objects). (b) The BL Lac $\mathrm{N}_{0.5}$ distribution (solid line) compared to that of FR II galaxies (dashed line; 6 objects). Two sources are hybrid and have been excluded.

\section{Results}

We compared the radio galaxies to BL Lacs studied by us previously (Pesce et al. 1995). In Figures $1 \mathrm{a}$ and $1 \mathrm{~b}$ we show the cumulative distribution of $\mathrm{N}_{0.5}$ values for BL Lacs and FR I sources and BL Lacs and FR II sources, respectively. A KS test gives the probability that the BL Lacs and FR I galaxies are from the same population as $91.4 \%$, slightly significant, while the probability for BL Lacs and FR II galaxies is only $63.3 \%$. We note the numbers are small, however, and need to be confirmed using the whole dataset. We are also analyzing $30 \mathrm{BL}$ Lacs with redshifts $<0.15$ to be compared directly to the radio galaxies.

Using CCD images we determined the incidence of close companions in our sample. Within a radius of $100 \mathrm{Kpc}$ the average number of companions in excess of the background is $1.1 \pm 1.6$. For FR I and II galaxies the numbers of excess companions are $1.2 \pm 1.8$ and $0.8 \pm 1.3$, respectively.

\section{References}

Bahcall, N.A. 1981, $A p J, 247,787$

Ekers, R.D., et al. 1989, MNRAS, 236, 737

Fasano, G., Falomo, R., \& Scarpa, R. 1995, MNRAS, in press

Hill, G.J. \& Lilly, S.J. 1991, ApJ, 367, 1

Kollgaard, R., et al. 1992, $A J$, 104, 1687

Lasker, B.M. et al 1990, $A J, 99,2019$

Owen, F.N., Ledlow, M.J., \& Keel, W.C. 1995, $A J, 109,140$

Pesce, J.E., Falomo, R., \& Treves, A. 1995, $A J, 110,1554$

Prestage, R.M., \& Peacock, J.A. 1988, MNRAS, 230, 131

Urry, C.M., \& Padovani, P. 1995, PASP, 107, 803

Valdes, F. 1982, in: Instrumentation in Astronomy IV, SPIE 331

Wall, J.V., \& Peacock, J.A. 1985, MNRAS, 216, 173

Yates, M.G., Miller, L., \& Peacock, J.A. 1989, MNRAS, 240, 129 